\title{
Time Domain Modeling of multimode selenide-chalcogenide glass fiber based mid infrared spontaneous emission sources
}

\author{
S. Sujecki1 ${ }^{1,2}$, Senior Member, IEEE , L. Sojka1 ${ }^{1}$ Z. Tang ${ }^{2}$, E. Barney ${ }^{2}$, \\ D. Furniss' ${ }^{2}$, T.M. Benson ${ }^{2}$, Senior Member, IEEE, A.B. Seddon ${ }^{2}$ \\ ${ }^{1}$ Department of Telecommunications and Teleinformatics, Faculty of Electronics, Wroclaw University of Science \\ and Technology, Wyb. Wyspianskiego 27, 50-370 Wroclaw, Poland \\ ${ }^{2}$ George Green Institute for Electromagnetics Research, The University of Nottingham, \\ University Park, NG7-2RD, Nottingham, UK \\ *Tel: (4871) 3204588, Fax: (4871) 322 3664, e-mail: Slawomir.Sujecki@pwr.edu.pl
}

\begin{abstract}
We develop time domain models of selenide-chalcogenide glass fiber based MIR spontaneous emission sources. The modeling parameters used are derived from experimentally obtained data. The models are based on the rate equations' approach to simulate the distribution of ions between the relevant energy levels. The optical power distribution within the fiber is calculated by solving a set of partial differential equations using specially developed finite difference schemes that allow for a direct inclusion of the step discontinuities appearing at the fiber facets. The results obtained allow for a thorough analysis of luminescence from lanthanide ion doped chalcogenide fibers.
\end{abstract}

Keywords: Mid infrared photonics, chalcogenide glass fibers, numerical modelling.

\section{INTRODUCTION}

Chalcogenide glass fiber technology is intensively developed due to its numerous applications, which use mid-infrared (MIR) light. A key element in MIR applications is the MIR light source. Thus chalcogenide glass fiber technology has progressed significantly over the last years [1-12]. So far chalcogenide glass fibers have been used to realize with success Raman lasers [13], supercontinuum sources [14] and spontaneous emission sources (SES) [15]. In this contribution a particular focus is given to the numerical analysis of the optical properties of SES operating within the MIR part of the optical spectrum, which have found application in sensor technology [16]. The structure considered consists of a section of a chalcogenide glass fiber, which is doped with lanthanide trivalent ions (Fig.1). A pump light source is applied at one end of the fiber whilst MIR light is collected from the other end. Modeling of such structures is closely related to the fiber laser modeling of chalcogenide glass based fiber laser structures. This topic has been very intensely researched using steady state models [17-20]. Here a time domain technique is developed for the purpose of the analysis, and numerical simulations of the time dependence of photon fluxes and level populations are performed.

\section{NUMERICAL MODELING}

The structure studied is doped with trivalent terbium ions (Fig.2). The pump is operating at $2950 \mathrm{~nm}$. The transition between levels 2 and 1 is assumed to be approximately non-radiative whilst the MIR light is generated by radiative transitions between levels 1 and 0 , which is approximately centered at $5000 \mathrm{~nm}$.

\section{MIR spontaneous emission}

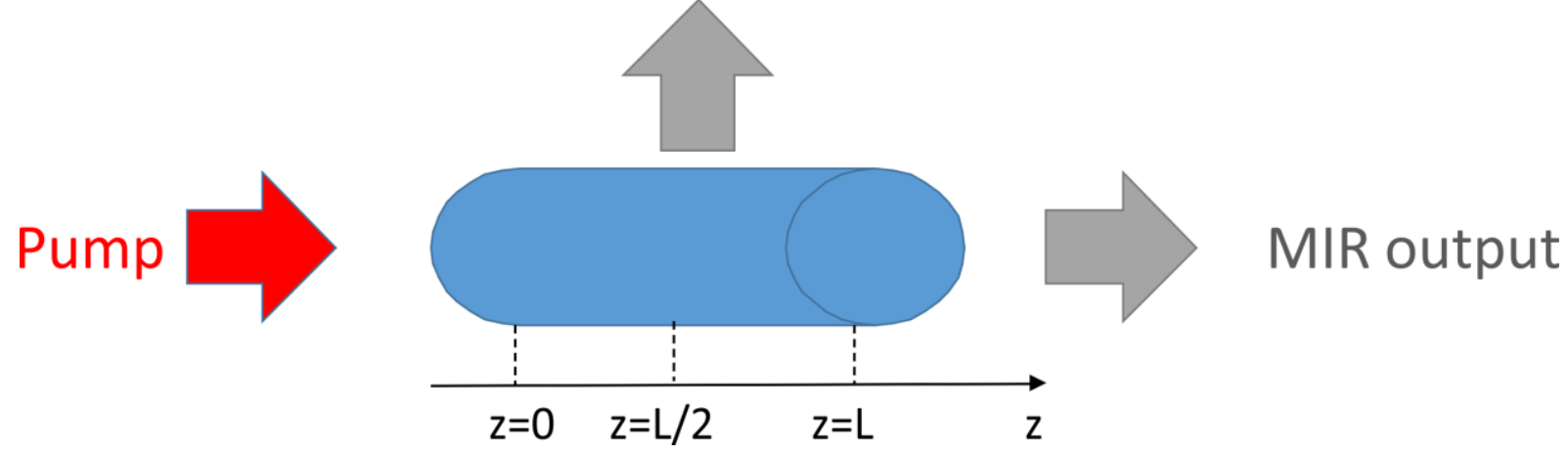

Figure 1.Schematic diagram of the spontaneous emission MIR light source.

The modeling algorithm applied, operates in three stages. In the first stage an initial guess is provided to a relaxation method steady state solver [21], which calculates a consistent solution at steady state for a given pump power. Then this solution is refined by the time domain model, which uses the method of lines [22] to refine the 
initial steady state solution. This avoids the generation of numerical artefacts, which could result from a numerical mismatch between the steady state solution calculated by the relaxation method and the time domain model. The last stage of the algorithm performs the time domain analysis for a give shape of the pump temporal evolution.

Fig. 3 shows a comparison between the spatial distributions of energy level populations and MIR and pump light within the fiber, which have been calculated by the algorithm at the first and second stage of the execution. The terbium ion concentration was $9.51 \times 10^{24} / \mathrm{m}^{3}$, the fiber length was $200 \mathrm{~mm}$ whilst the incident pump power was $100 \mathrm{~mW}$. These results confirm a very good agreement between the results obtained by two different numerical techniques.

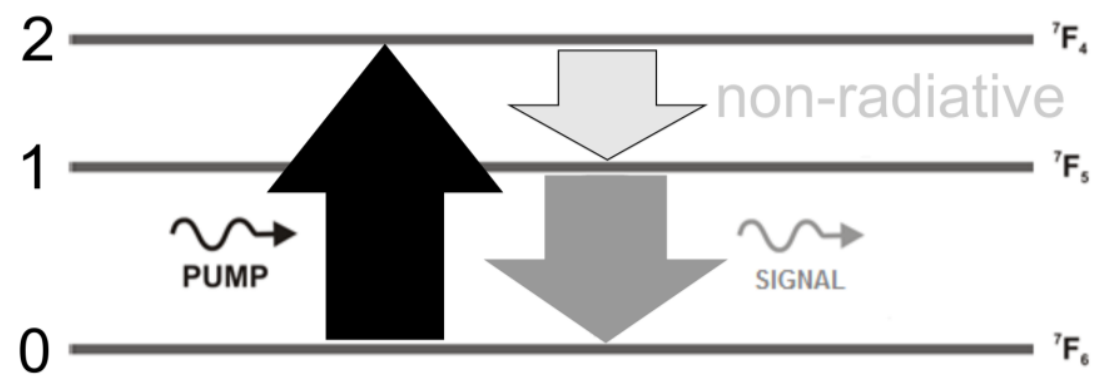

Figure 2. A schematic diagram of trivalent terbium ion energy levels.
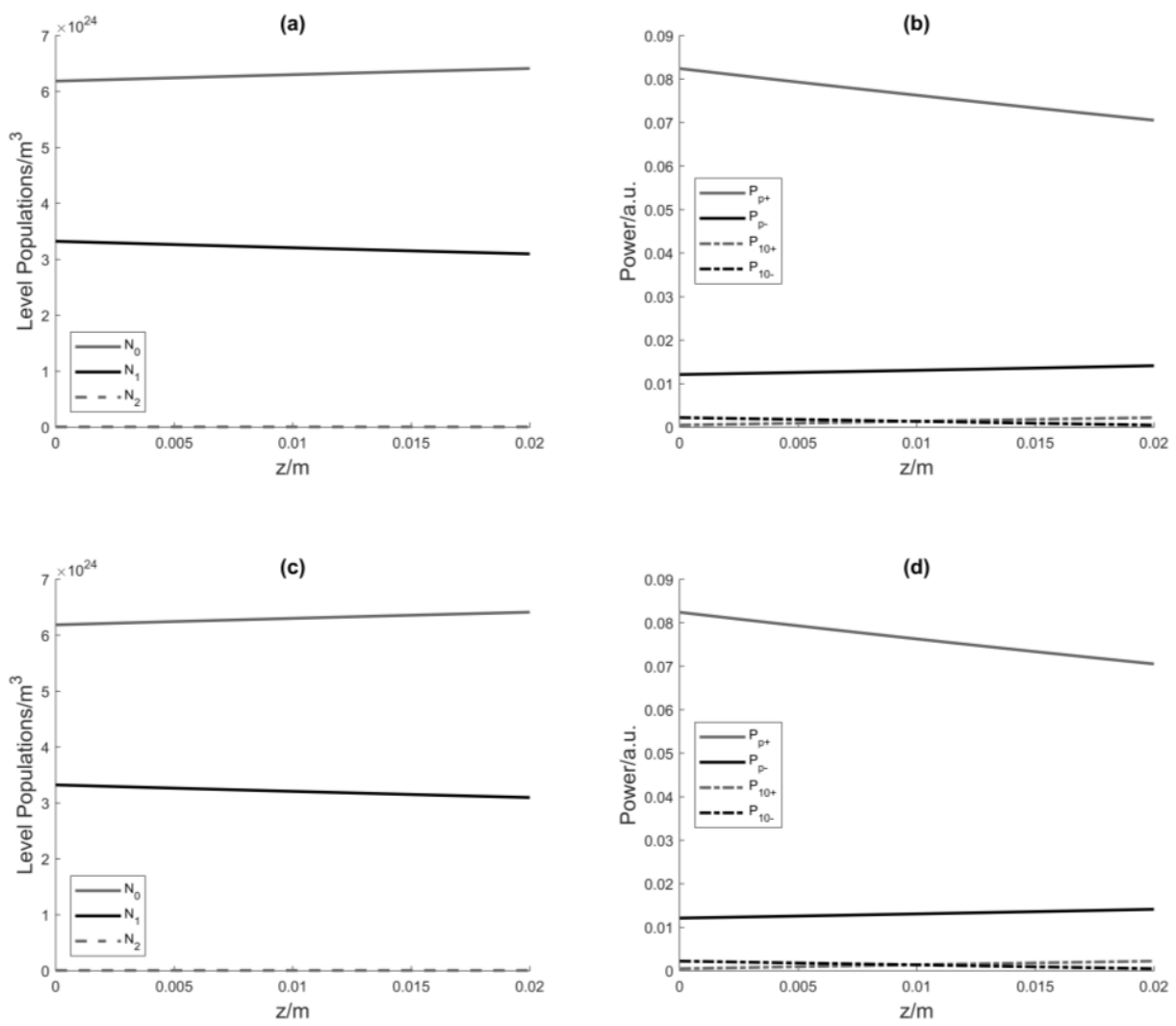

Figure 3. Spatial dependence of the level populations and power distributions calculated by the relaxation method (a) and (b), respectively, and the time domain method (c) and (d), respectively. The superscripts ' + ' and '- correspond to forward and backward propagating wave, respectively.

Fig. 4 shows the dependence of energy level populations and signal and pump powers on the spatial and temporal dimensions. The pump power was switched off from a high level of $100 \mathrm{~mW}$ along a cosine square temporal waveform within $0.01 \mathrm{~ms}$. The level 1 population at $\mathrm{z}=0$ (a) and $\mathrm{z}=\mathrm{L}$ (b) follows a slow decay at a rate governed by the radiative lifetime of level 1 . Since, the population of level 3 is near to zero the slow decay of level 1 population is reflected in the corresponding growth of the level 0 population. The pump power for the forward traveling wave at $\mathrm{z}=\mathrm{L}$ and the backward traveling wave at $\mathrm{z}=0$ follows the shape of the switching of 
cosine square waveform with a tiny delay resulting from the photon lifetime within the cavity (Fig.4c). The signal forward and backward traveling waves decay at a much slower rate, and follow the decay rate of the energy level 1 population. At the final simulation time equal to $100 \mathrm{~ms}$ a steady state is reached approximately, whereby almost all ions are in the ground state (Fig.4e). There is still small residual photon population within the cavity (Fig.4f) due to a relatively low decay time of $13.1 \mathrm{~ms}$.
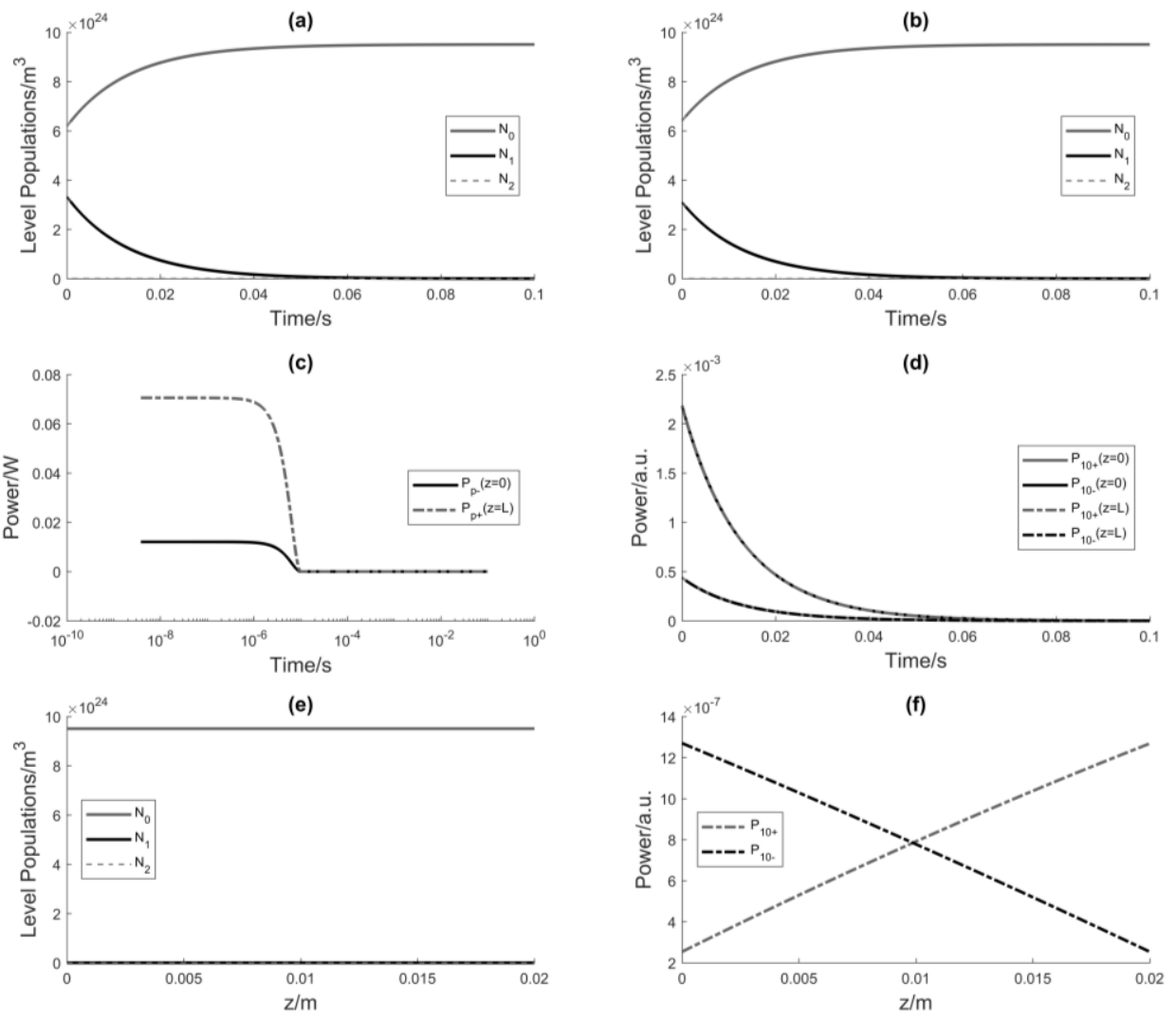

Figure 4. Spatial distribution and temporal dependence of level populations and power distributions calculated by the time domain model: (a) - energy level populations at $z=0,(b)$ - energy level populations at $z=L,(c)-$ power temporal dependence, $(d)$ - MIR signal temporal dependence, (e)-energy level distributions at $100 \mathrm{~ms}$,

(f) - MIR signal distribution at $100 \mathrm{~ms}$. The superscripts '+' and '- 'correspond to forward and backward propagating wave, respectively.

\section{CONCLUSIONS}

In the paper a time domain model of terbium ion doped selenide-chalcogenide glass fiber is used to model the time and spatial dependence of the energy level populations and photon densities. The results obtained give a detailed picture of the spatio-temporal dynamics of photons within the fiber and of the interactions between photons and ions.

\section{ACKNOWLEDGEMENTS}

This project has received funding from the European Union's Horizon 2020 research and innovation programme under the Marie Skłodowska-Curie grant agreement No. 665778 (National Science Centre, Poland, Polonez

Fellowship 2016/21/P/ST7/03666)

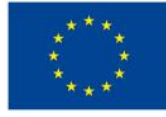

Science and Higher Education under the project entitled "Iuventus Plus", 2016-2018 (project no.

0441/IP2/2015/73). 


\section{REFERENCES}

[1] M. F. Churbanov et al., "Chalcogenide glasses doped with Tb, Dy and Pr ions," Journal of NonCrystalline Solids, vol. 326, pp. 301-305, Oct 12003.

[2] E. V. Karaksina, V. S. Shiryaev, M. F. Churbanov, E. A. Anashkina, T. V. Kotereva, and G. E. Snopatin, "Core-clad $\operatorname{Pr}\left({ }^{3+}\right)$-doped Ga(In)-Ge-As-Se-(I) glass fibers: Preparation, investigation, simulation of laser characteristics," Optical Materials, vol. 72, pp. 654-660, Oct 2017.

[3] G. E. Snopatin, V. S. Shiryaev, V. G. Plotnichenko, E. M. Dianov, and M. F. Churbanov, "High-purity chalcogenide glasses for fiber optics," Inorganic Materials, vol. 45, no. 13, pp. 1439-1460, Dec 2009.

[4] Z. Tang et al., "Low loss Ge-As-Se chalcogenide glass fiber, fabricated using extruded preform, for mid-infrared photonics," Optical Materials Express, vol. 5, no. 8, pp. 1722-1737, Aug 12015.

[5] H. Sakr et al., "Superior photoluminescence (PL) of $\mathrm{Pr}^{3+}-\mathrm{In}$, compared to $\mathrm{Pr}^{3+}-\mathrm{Ga}$, selenidechalcogenide bulk glasses and PL of optically-clad fiber," Optics Express, vol. 22, no. 18, pp. 2123621252, Sep 82014.

[6] A. B. Seddon, Z. Tang, D. Furniss, S. Sujecki, and T. M. Benson, "Progress in rare-earth-doped midinfrared fiber lasers," Optics Express, vol. 18, no. 25, pp. 26704-26719, Dec 62010.

[7] Z. Tang, N. C. Neate, D. Furniss, S. Sujecki, T. M. Benson, and A. B. Seddon, "Crystallization behavior of Dy ${ }^{3+}$-doped selenide glasses," Journal of Non-Crystalline Solids, vol. 357, no. 11-13, pp. 2453-2462, Jun 2011.

[8] Z. Q. Tang et al., "Mid-infrared photoluminescence in small-core fiber of praseodymium-ion doped selenide-based chalcogenide glass," Optical Materials Express, vol. 5, no. 4, pp. 870-886, Apr 2015.

[9] J. Ari et al., "Rare-earth doped chalcogenide glasses for mid-IR gas sensor applications," in Optical Components and Materials Xiv, vol. 10100, S. Jiang and M. J. F. Digonnet, Eds. (Proceedings of SPIE, 2017.

[10] V. Moizan et al., "Er ${ }^{3+}$-doped GeGaSbS glasses for mid-IR fibre laser application: Synthesis and rare earth spectroscopy," Optical Materials, vol. 31, no. 1, pp. 39-46, Sep 2008.

[11] J. S. Sanghera, L. B. Shaw, and I. D. Aggarwal, "Chalcogenide glass-fiber-based mid-IR sources and applications," Ieee Journal of Selected Topics in Quantum Electronics, vol. 15, no. 1, pp. 114-119, JanFeb 2009.

[12] L. B. Shaw, B. Cole, P. A. Thielen, J. S. Sanghera, and I. D. Aggarwal, "Mid-wave IR and long-wave IR laser potential of rare-earth doped chalcogenide glass fiber," Ieee Journal of Quantum Electronics, vol. 37, no. 9, pp. 1127-1137, Sep 2001.

[13] M. Bernier, V. Fortin, M. El-Amraoui, Y. Messaddeq, and R. Vallee, "3.77 mu m fiber laser based on cascaded Raman gain in a chalcogenide glass fiber," Optics Letters, vol. 39, no. 7, pp. 2052-2055, Apr 2014.

[14] C. R. Petersen et al., "Mid-infrared supercontinuum covering the 1.4-13.3 mu m molecular fingerprint region using ultra-high NA chalcogenide step-index fibre," Nature Photonics, vol. 8, no. 11, pp. 830834, Nov 2014.

[15] A. L. Pele et al., "Dy3+ doped GeGaSbS fluorescent fiber at $4.4 \mathrm{mu}$ m for optical gas sensing: Comparison of simulation and experiment," Optical Materials, vol. 61, pp. 37-44, Nov 2016.

[16] F. Starecki et al., "Mid-IR optical sensor for CO2 detection based on fluorescence absorbance of $\mathrm{Dy}^{3+}:$ Ga5Ge20Sb10S65 fibers," Sensors and Actuators B-Chemical, vol. 207, pp. 518-525, Feb 2015.

[17] S. Sujecki et al., "Modelling of a simple Dy ${ }^{3+}$ doped chalcogenide glass fibre laser for mid-infrared light generation," Optical and Quantum Electronics, vol. 42, no. 2, pp. 69-79, Jan 2010.

[18] L. Sojka et al., "Study of mid-infrared laser action in chalcogenide rare earth doped glass with Dy ${ }^{3+}$, $\mathrm{Pr}^{3+}$ and $\mathrm{Tb}^{3+}$," Optical Materials Express, vol. 2, no. 11, pp. 1632-1640, Nov 12012.

[19] M. C. Falconi et al., "Dysprosium-doped chalcogenide master oscillator power amplifier (MOPA) for mid-IR emission," Journal of Lightwave Technology, vol. 35, no. 2, pp. 265-273, Jan 2017.

[20] M. C. Falconi et al., "Design of an efficient pumping scheme for mid-IR Dy ${ }^{3+}: \mathrm{Ga}_{5} \mathrm{Ge}_{20} \mathrm{Sb}_{10} \mathrm{~S}_{65} \mathrm{PCF}$ fiber laser," Ieee Photonics Technology Letters, vol. 28, no. 18, pp. 1984-1987, Sep 2016.

[21] S. Sujecki et al., "Numerical modelling of $\mathrm{Tb}^{3+}$ doped selenide-chalcogenide multimode fibre based spontaneous emission sources," Optical and Quantum Electronics, vol. 49, no. 12, Dec 2017, Art. no. 416.

[22] S. Sujecki, "Simple and efficient method of lines based algorithm for modeling of erbium doped Qswitched fluoride fiber lasers," Journal of the Optical Society of America B-Optical Physics, vol. 33, no. 11, pp. 2288-2295, Nov 2016. 